\title{
Three-dimensional particle trajectories and waste beam losses in injection dump beam line of Spallation Neutron Source accumulation ring
}

\author{
J. G. Wang* and M. A. Plum \\ Oak Ridge National Laboratory, Oak Ridge, Tennessee 37831, USA
}

(Received 28 August 2007; published 4 January 2008)

\begin{abstract}
The Spallation Neutron Source ring injection dump beam line has been suffering high beam losses since its commissioning. In order to understand the mechanisms of the beam losses, we have performed 3D simulation studies of the beam line. The 3D models consist of three injection chicane dipoles and one injection dump septum magnet. The 3D particle trajectories in the models are computed. We then extend particle optics calculations to the injection dump. Our studies have clearly shown some design and operation problems, which cause beam losses in the injection dump beam line. These include incorrect chicane dipole settings, incorrect position of a chicane dipole, too small aperture of injection dump septum, and inadequate focusing downstream. This paper reports our findings and the remedies to the injection beam loss problems.
\end{abstract}

DOI: 10.1103/PhysRevSTAB.11.014002

PACS numbers: 29.27.Ac, 29.27.Eg, 41.85.Ar, 41.85.Lc

\section{INTRODUCTION}

The Spallation Neutron Source (SNS) accelerator employs non-Liouvillian injection $[1,2]$ to obtain high intensity proton beams in its accumulator ring. As shown in Fig. 1, a $1 \mathrm{GeV} \mathrm{H}^{-}$beam from the linac is focused on a primary foil $(\mathrm{F} 1)$ in the accumulator ring to produce a proton beam through charge exchange. A small portion of the $\mathrm{H}^{-}$particles is not fully stripped by $\mathrm{F} 1$ and becomes $\mathrm{H}^{0}$ particles. The probability that the injected $\mathrm{H}^{-}$particles pass through the foil and remain as $\mathrm{H}^{-}$particles is negligibly small, but some incoming $\mathrm{H}^{-}$particles may miss the foil. The $\mathrm{H}^{0}$ and $\mathrm{H}^{-}$particles after $\mathrm{F} 1$ propagate forward through chicane dipole D3 and strike a secondary foil (F2), after which they all should be fully stripped to proton particles. These particles are the so-called waste beams that are bent by a chicane dipole D4 and are then transported through an injection dump septum magnet (IDSM). The IDSM is a magnetic dipole with significant gradient component, as explained later. It not only bends the two waste beams further away from the proton beam line, but also provides horizontal focusing for each of them. After IDSM the waste beams go through a horizontally defocusing quadrupole magnet and are then transported to an injection dump (IDump). The waste beams are designated as $\mathrm{H}^{-}$-proton beam and $\mathrm{H}^{0}$-proton beam according to their origin. The whole system behaves like a particle spectrometer for charge separation.

Since the SNS ring commissioning the entire injection dump beam line, especially IDSM, has been suffering high beam losses. After exhausting all of the operational means, the beam losses still remain. This limits the ramp-up of the SNS ring beam power. In order to understand why and where the waste beam particles are lost, we have performed $3 \mathrm{D}$ modeling of the injection dump beam line consisting of

\footnotetext{
*jgwang@ornl.gov
}

three chicane dipoles and IDSM. The particle trajectories in the models and the particle optics further downstream are calculated. The study has clearly shown the waste beam loss mechanisms and has led to the remedies to the problems.

In Sec. II, we present the injection constraints, which dictate the chicane dipole settings. They are critical to the waste beam transport, as well as to obtaining good injection and a good equilibrium orbit (closed orbit) for the circulating proton beam. The conditions for transporting the waste beams through IDSM are also discussed. The 3D simulation models of the waste beam line are presented in Sec. III. The magnet performance and field distributions are briefly described, and the initial conditions for particle tracking are also discussed. Section IV is devoted to the 3D particle trajectories starting from the primary foil (F1) through IDSM and ending at the entrance of the quadrupole magnet downstream. The waste beam particle losses in both the $x-z$ and $y-z$ planes due to different mechanisms are demonstrated. In Sec. V, we analyze the particle optics through the quadrupole magnet down to the injection dump. It is shown that a single quadrupole magnet is not adequate to transport the waste beam down to the dump. Section VI contains the conclusions of our study, the remedies to the problems, and experimental verification of the 3D models. A short summary of this work can be found in Sec. VII.

\section{INJECTION CONSTRAINTS}

The SNS ring was designed and fabricated at Brookhaven National Lab (BNL) [3]. Its injection system is integrated into one of four straight sections of the ring. As shown in Fig. 1, four chicane dipoles D1-D4 create a static orbit bump. Four kickers (not shown in the figure) in each of the transverse dimensions generate dynamic bumps for painting particles in both the $x$ and $y$ directions. The 


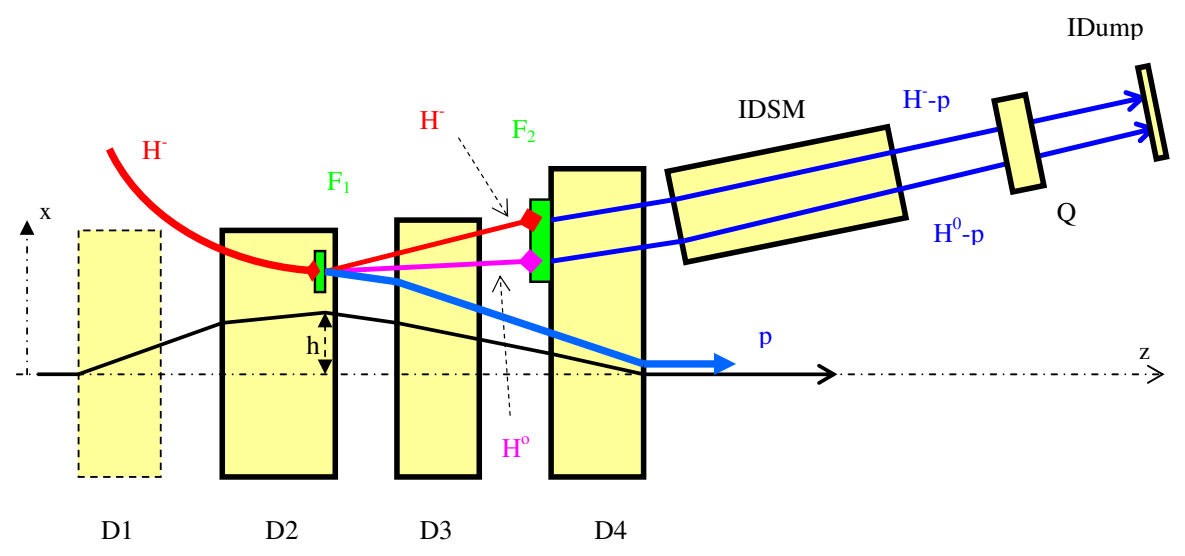

FIG. 1. (Color) Injection dump beam line schematic.

chicane dipole settings are critical for obtaining good injection and a good equilibrium orbit of the circulating proton beam, as well as for good transport of the waste beams. As shown in Table I, there exist mainly three chicane dipole settings for the SNS ring. The design setting $[4,5]$ and the delivered setting [6,7] were developed during the early stage of the project and overlooked some injection constraints as described below. The production setting [8] was worked out during the ring commissioning to yield good injection and a good equilibrium orbit for the circulating proton beam, and has been employed in operation ever since. We have studied all three settings in our 3D models for the waste beam transport.

\section{A. Closed orbit bump and good injection}

The most important condition for setting the four injection chicane dipoles is to make local closure of the injection orbit bump. This results in a good equilibrium orbit for the circulating proton beam. A proton entering D1 on the $z$-axis of the straight section, passing through the orbit bump, should exit from D4 on the $z$-axis. Intuitively, the first condition is that the algebraic sum of the four dipole bending angles equals zero, i.e.

$$
\theta_{1}+\theta_{2}+\theta_{3}+\theta_{4}=0 \text {. }
$$

A dipole bending angle is measured by the rotation angle between the incident particle trajectory and the exiting particle trajectory before and after the dipole. Our convention is that the bending angles are positive when the proton is bent counterclockwise horizontally if one looks down from above. Here, $\theta_{1}$ and $\theta_{4}$ are positive, while $\theta_{2}$

TABLE I. Chicane dipole bending angles (mrad).

\begin{tabular}{lcccc}
\hline \hline Chicane dipole setting & D1 & D2 & D3 & D4 \\
\hline Design [4,5] & 42.0 & -46.2 & -42.0 & 46.2 \\
Delivered [6,7] & 42.0 & -53.1 & -35.6 & 46.2 \\
Production [8] & 42.0 & -53.1 & -28.3 & 39.4 \\
\hline \hline
\end{tabular}

and $\theta_{3}$ are negative. The condition (1) guarantees that the proton goes through the orbit bump without gaining any transverse velocity in the $x$-direction. In addition, the proton also should not develop any offset from the $z$-axis at the D4 exit. This can be accomplished if, to the first-order approximation, the following condition is satisfied:

$$
\left(d_{12}+d_{23}+d_{34}\right) \theta_{1}+\left(d_{23}+d_{34}\right) \theta_{2}+d_{34} \theta_{3}=0 .
$$

Here $d_{12}$ is the distance between D1 and D2 centers, etc.

To achieve good injection and painting, the incoming $\mathrm{H}^{-}$beam and a part of the circulating proton beam should merge at the primary foil with zero relative angles. If the injection takes place at a position where the incoming $\mathrm{H}^{-}$ beam is parallel with the $z$-axis, as designed, the primary foil should be located at the maximum orbit bump. Then, good injection can be achieved by the following condition:

$$
\theta_{2}=-\left(1+\frac{1}{R}\right) \theta_{1}
$$

where $R$ is the ratio of the D2 field integrals upstream and downstream of the primary foil, and is determined by the D2 field distribution. The static orbit bump amplitude is another important parameter for injection and painting. In this case, the orbit bump amplitude $h$ can be found, for small angle approximation, as

$$
h=\left(d_{12}-\frac{d_{2 F}}{R}\right) \theta_{1},
$$

where $d_{2 F}$ is the distance between the D2 center and the primary foil.

Given all the mechanical positions, the orbit bump height $h$, and the ratio $R$ of $\mathrm{D} 2$, the four conditions above uniquely determine the four bending angles. In the SNS ring injection section, $d_{12}=238.1, d_{23}=181.4, d_{34}=$ $202.9, d_{2 F}=30.71 \mathrm{~cm}$, and the D2 field integral ratio is measured to be $R=3.784$. If we choose the $\mathrm{D} 1$ bending angle $\theta_{1}=42.0 \mathrm{mrad}$ as from the BNL/SNS data, condition (4) leads to the orbit bump amplitude $h=9.66 \mathrm{~cm}$. Conditions (3), (2), and (1) yield the dipole bending angles 
$\theta_{2}=-53.1, \theta_{3}=-28.3$, and $\theta_{4}=39.4 \mathrm{mrad}$, respectively. This is exactly the same as the production setting in Table I. We can also see that the delivered setting does not satisfy condition (1), leading to an unclosed orbit bump with about $1.51 \mathrm{~cm}$ offset at the D4 exit.

Another important consideration for the $\mathrm{H}^{-}$beam injection is the collection of stripped electrons. The primary foil is located in the D2 downstream fringe region, where the dipole field $B_{y}$ is about $2.5 \mathrm{kG}$ and the field angle $\left[\operatorname{Atan}\left(-B_{z} / B_{y}\right)\right]$ is more than $200 \mathrm{mrad}$. Thus, the stripped electrons, leaving the primary foil at the speed of the incoming $\mathrm{H}^{-}$particles, rotate with a Larmor radius of more than $1.2 \mathrm{~cm}$. They should clear the lower edge of the foil on their first turn, and spiral down to a water-cooled carbon collector below [4]. The design setting in Table I requires a D2 field integral ratio $R=10$ to satisfy Eq. (3). Therefore, the primary foil should be moved further away downstream, at which $(z=47.3 \mathrm{~cm}$ instead of $30.71 \mathrm{~cm})$ the dipole field in the as-built magnet is only about $1.54 \mathrm{kG}$. The stripped electrons would be trapped in the magnetic field. Thus, the design setting is not applicable. This problem was discovered after the magnets were made and measured. The delivered setting was introduced then, but unfortunately, it does not satisfy the closed orbit bump requirement. This leads to large displacement and unstable oscillation about the displaced equilibrium orbit of the circulating proton beam, and thus, to high beam losses in the ring.

\section{B. Transport of waste beams through IDSM}

Survey data [9] show that the downstream quadrupole magnet axis and the $z$-axis in the ring straight section form an angle of $\theta_{T}=12.57^{\circ}$ (219.4 mrad). For a reference $\mathrm{H}^{0}$-proton particle, which is initially in parallel with the $z$-axis and is bent by D4 first and then by IDSM, the following condition should be satisfied:

$$
\theta_{T} \leq \theta_{4}+\theta_{s 0}<\theta_{T}+\delta \theta .
$$

Here $\theta_{s 0}$ is the IDSM bending angle for the $\mathrm{H}^{0}$-proton particle, which will be explained in more detail in Sec. III B. $\delta \theta$ is a positive, small open angle from the downstream quadrupole magnet entrance to the $\mathrm{H}^{0}$-proton exit at IDSM. The distance between the IDSM exit face and the quadrupole magnet center is about $6.25 \mathrm{~m}$. Suppose the reference particle exits IDSM at a transverse distance of $8 \mathrm{~cm}$ from its axis, we would have a roughly estimated $\delta \theta \sim 13 \mathrm{mrad}$. The unequal sign is used in Eq. (5a) because it is desired that the reference $\mathrm{H}^{0}$-proton particle converges toward the axis of the downstream quadrupole magnet. With the production setting of the chicane dipoles, D4 would bend the $\mathrm{H}^{0}$-proton particles by $39.4 \mathrm{mrad}$. An additional bending angle of a little more than $180.0 \mathrm{mrad}$ from IDSM would be needed for a reference $\mathrm{H}^{0}$-proton particle.
By the same token, an $\mathrm{H}^{-}$-proton particle would be first bent by the fields between F1 and F2 plus the D4 field, and then by IDSM. This leads to the following condition:

$$
\theta_{T}-\delta \theta<-\frac{\theta_{2}}{1+R}-\theta_{3}+\theta_{4}+\theta_{s^{-}} \leq \theta_{T}
$$

where $\theta_{s^{-}}$is the IDSM bending angle for a reference $\mathrm{H}^{-}$-proton particle. Note that $\theta_{s^{-}} \neq \theta_{s 0}$ because IDSM is a gradient dipole magnet as described later. With the production setting for the chicane dipoles, the fields between $\mathrm{F} 1$ and $\mathrm{F} 2$ yield a bending angle of about $38.9 \mathrm{mrad}$. An additional bending angle of a little less than $141.1 \mathrm{mrad}$ from IDSM would be needed for a reference $\mathrm{H}^{-}$-proton particle. Though we use the same notation of the chicane bending angles in Eqs. (5a) and (5b) as that in Eqs. (1)-(4), their numerical values may be slightly different due to different paths considered.

As described in Sec. II A, the bending angles of the four chicane dipoles are uniquely determined by Eqs. (1)-(4). Thus, Eqs. (5a) and (5b) have to be fulfilled by the IDSM bending angles $\theta_{s 0}$ and $\theta_{s^{-}}$. But, it is not trivial to satisfy the two equations simultaneously by one septum magnet. It requires certain trajectory positions in the device, which are determined by the initial waste beam positions and angles at the IDSM entrance. In most cases, the two waste beams incident to the IDSM entrance face are not at normal direction to the IDSM face. Especially for the $\mathrm{H}^{-}$-proton particles, which are always incident to the IDSM entrance with large face-rotation angles due to additional bending by the fields between F1 and F2. This makes it very difficult to predict their trajectories inside IDSM without 3D modeling.

\section{3D SIMULATION MODELS}

The design of the SNS ring injection waste beam line was based on 2D calculations, where the particle motion in the $y$-direction was overlooked, resulting in a very small vertical aperture of IDSM. In addition, each magnet on the beam line was treated as a conventional hard-edge component, where the effect of magnetic fringe field and interferences were ignored. This was in principle inaccurate.

We believe that 3D simulation models and 3D particle trajectories are necessary to take into account all three discrete magnetic field components $B_{y}, B_{x}$, and $B_{z}$ in beam dynamics. The chicane dipoles D2, D3, D4, and IDSM are all large aperture magnets. They produce significant fringe fields, which affect particle motions. This effect can be automatically included in 3D analyses. Further, the four magnets are closely packed and their fields are overlapping. The magnetic interference changes the field distributions in between any two magnets. Thus, good 3D models should include all the four magnets. 


\section{A. Simulation models}

The simulation environment employed in this study is OPERA3D/TOSCA v.11 [10]. The models consist of four magnets, i.e., D2, D3, D4, and IDSM as shown in Fig. 2, where three particle trajectories are also illustrated. The simulation models are built by the OPERA package "Modeller" rather than the "Pre-Processor". The Modeller makes it much easier to simulate two or more magnets together.

The coordinate system origin is at the D2 mechanical center and the units are in centimeters. The mechanical centers of D3 and D4 are at $(-0.772,0,181.4)$ and $(-9.155,0,384.3)$; the center of the IDSM entrance face is at $(19.40,2.3,493.1)$, about which the magnet is rotated by $2.613^{\circ}$. The coordinates of the two foil centers are $(3.997,2.3,30.71)$ and $(7.29,2.3,293.5)$. All the mechanical dimensions are taken from the BNL design parameters and are verified by survey and alignment data [9]. Note that the two foil centers are $2.3 \mathrm{~cm}$ above the midplanes of the three chicane dipoles. The D4 axis is offset from the D2 axis by $-9.155 \mathrm{~cm}$ in the $x$-direction. These component locations have significant consequences for particle motion.

We have studied all three sets of chicane dipole settings in different 3D models and have compared their performance. The preliminary investigation of the waste beam losses in a model with the delivered setting was reported before [11]. In this paper we report the results only from the production setting. This setting has been employed since the ring commissioning and typically shows all the waste beam loss mechanisms [12]. In the production setting the current amplitudes of chicane dipoles D2 and D3 are 2126 and 1449 A, while they are 1737 and $2914 \mathrm{~A}$ in D4 and IDSM, respectively. These currents in D2, D3, and D4 yield bending angles of $-53.1,-28.3$, and $39.4 \mathrm{mrad}$ for a $1 \mathrm{GeV}$ proton beam. Chicane dipole D1, which is not a part of the waste beam line and is not included in the models, has a bending angle of $42.0 \mathrm{mrad}$ in all the three chicane settings.

A great difficulty with these 3D simulation models is their very large volumes, which present a significant challenge in mesh generations. All the cell properties must be carefully assigned and adjusted. A number of artificial cuts must be inserted in the models in order to make fine meshes. A model typically contains about $14 \times 10^{6}$ total elements, which is close to the maximum allowed by the software [13]. Other model statistics include $8.3 \times 10^{6}$ total nodes, $16 \times 10^{6}$ edges, $9 \times 10^{6}$ linear tetrahedral, $5 \times 10^{6}$ quadratic tetrahedral, and $8 \times 10^{6}$ equations. The TOSCA solutions yield a postprocessor file of 4.132 GB for each setting, from which we obtain the field distributions and $3 \mathrm{D}$ particle trajectories.

\section{B. Magnets and fields on beam line}

All the magnets for the SNS ring injection system and its waste beam line were designed and developed by the BNL/ SNS team. A great effort was devoted to the modeling, design optimization, and measurements of these devices at BNL. We have also performed 3D simulations of these magnets with OPERA-3D/TOSCA, and obtained quite good agreement with the results reported by BNL. The main design features of these magnets and some field distributions are described below. This helps to understand the waste beam line operation.

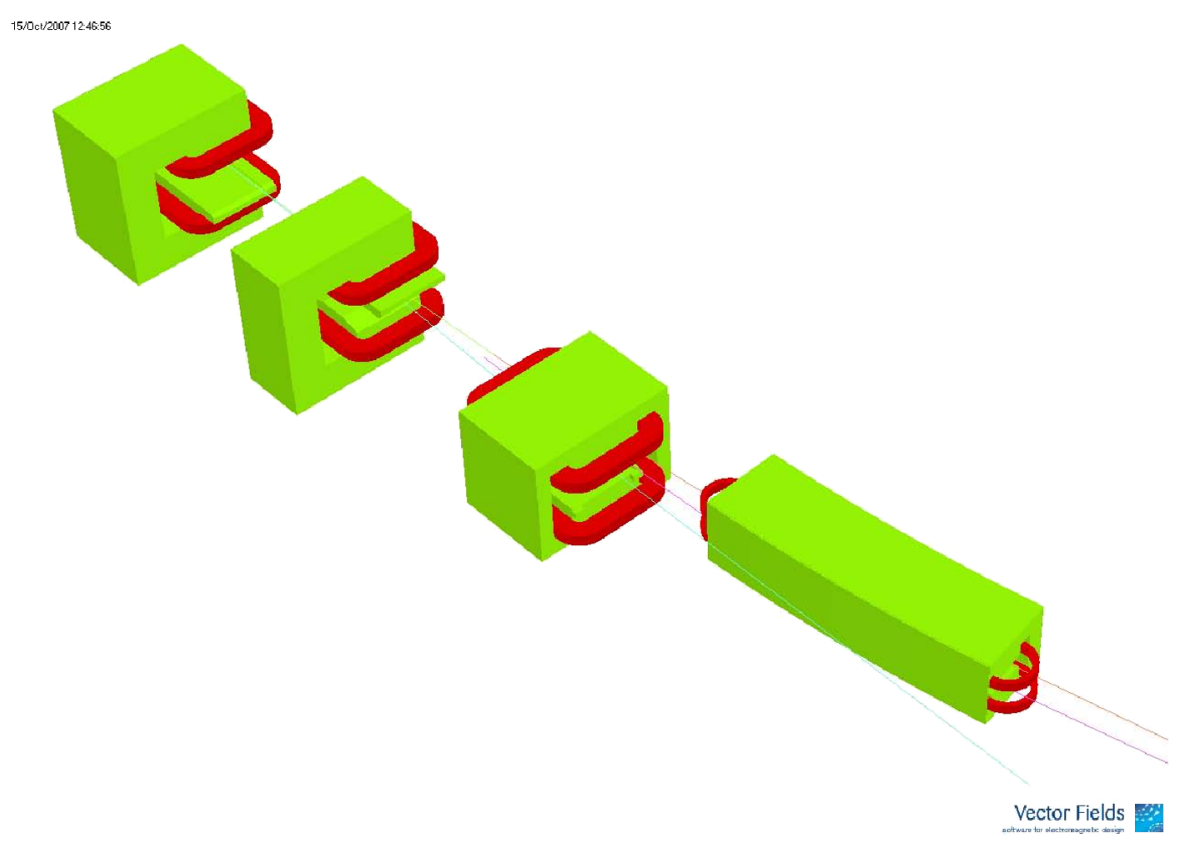

FIG. 2. (Color) 3D simulation model (from left to right: D2, D3, D4, and IDSM). 


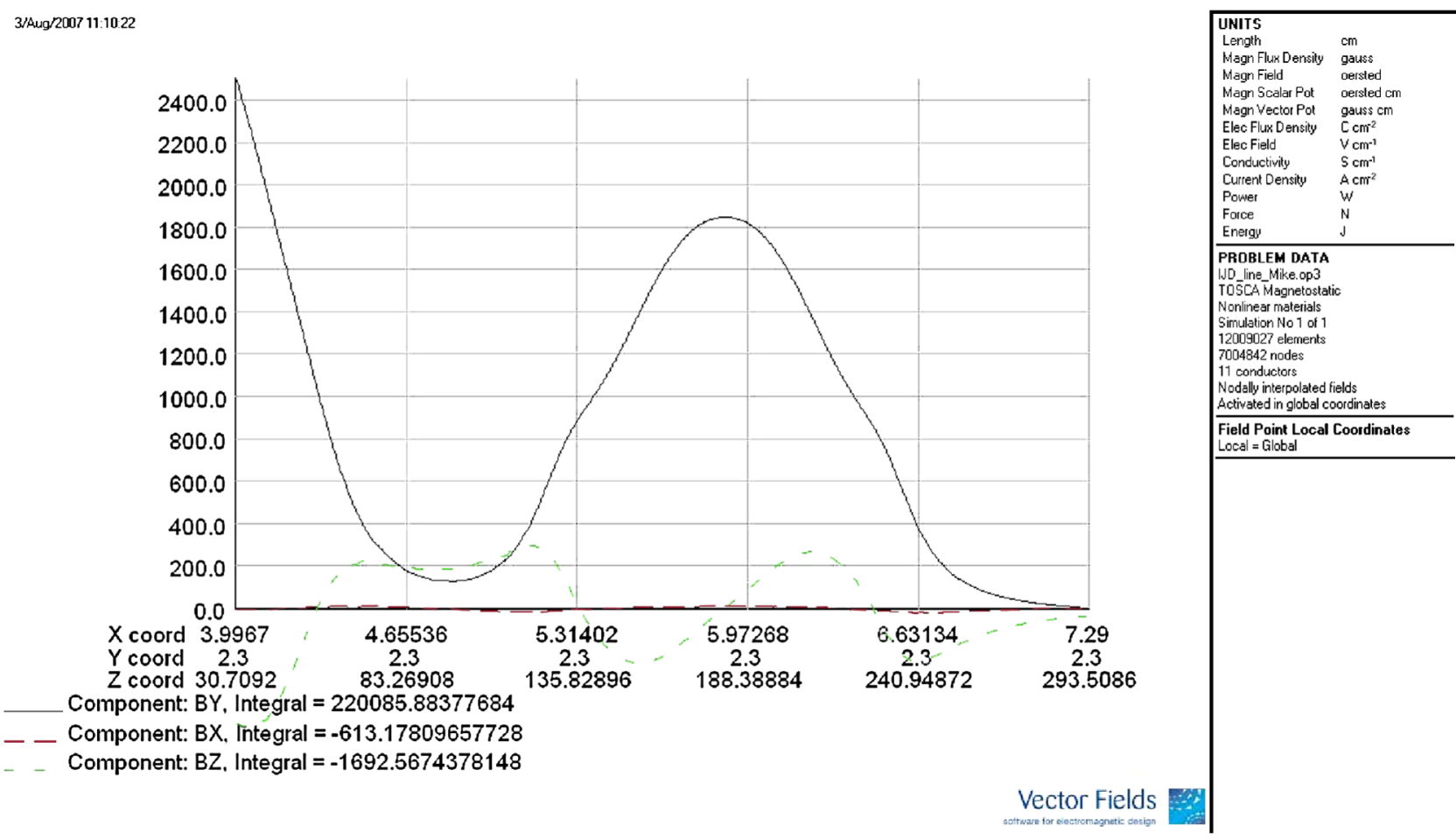

FIG. 3. (Color) Magnetic field along a line from F1 center to $\mathrm{F} 2$ center, where the units are centimeter for $x, y, z$ coordinates and gauss for magnetic flux density.

Chicane dipoles D2 and D3 have been studied extensively in 3D simulations and experimental measurements $[6,7,14,15]$. These dipoles are C-shaped magnets with complementary pole tips. The two dipoles have no symmetric bending planes, thus the magnetic field is three dimensional everywhere. The primary foil (F1) is located around the D2 downstream edge, where the dipole field $B_{y}$ is $2.5 \mathrm{kG}$. The field tilt $\left[\operatorname{Atan}\left(-B_{z} / B_{y}\right)\right]$ at $\mathrm{F} 1$ is about $220 \mathrm{mrad}$. The ratio of the D2 field integrals upstream and downstream of the foil is $R=3.784$. The peak field of D3 is less than $1.9 \mathrm{kG}$. The secondary foil (F2) is located downstream of dipole D3. Figure 3 shows the field distribution along a straight line from the F1 center $(3.997,2.3$, $30.71)$ to the $\mathrm{F} 2$ center $(7.29,2.3,293.5)$. The $B_{y}$ integral is $0.2201 \mathrm{~T} \mathrm{~m}$, yielding a total bending angle of $38.9 \mathrm{mrad}$ for a $1 \mathrm{GeV} \mathrm{H}^{-}$particle.

Chicane dipole D4 is a typical H-shaped magnet $[15,16]$. The dipole bends both the circulating beam and the waste beams, so it should have a large horizontal aperture with good field quality. The pole tip is $51.82 \mathrm{~cm}$ wide. In order to improve the field quality, the pole tips have longitudinal shims on their edges, and the magnet also contains eight $z$-bumps to enhance its field in the region far from its center. Though the dipole has midplane symmetry, most waste beam particles enter the magnet already above the symmetry plane. Thus, the fields for all the reference trajectories are three dimensional.

The IDSM [15-17] is a sector dipole with significant quadrupole components. Its curved pole tips are $193 \mathrm{~cm}$ in length and $26.52 \mathrm{~cm}$ in width. The septum handles both $\mathrm{H}^{0}$-proton and $\mathrm{H}^{-}$-proton waste beams with their trajectories going through the two sides of its longitudinal axis. The vertical aperture of its vacuum chamber is as small as $\pm 2.07 \mathrm{~cm}$. Though the magnet has up-down symmetry, the most waste beam particles coming from D4 are already above the symmetry plane. Figure 4 shows the magnetic field distribution on the midplane across the IDSM gap at its longitudinal center. The two vertical, dashed lines indicate the pole-tip width boundaries, while the two solid lines depict the relative positions of the vacuum chamber

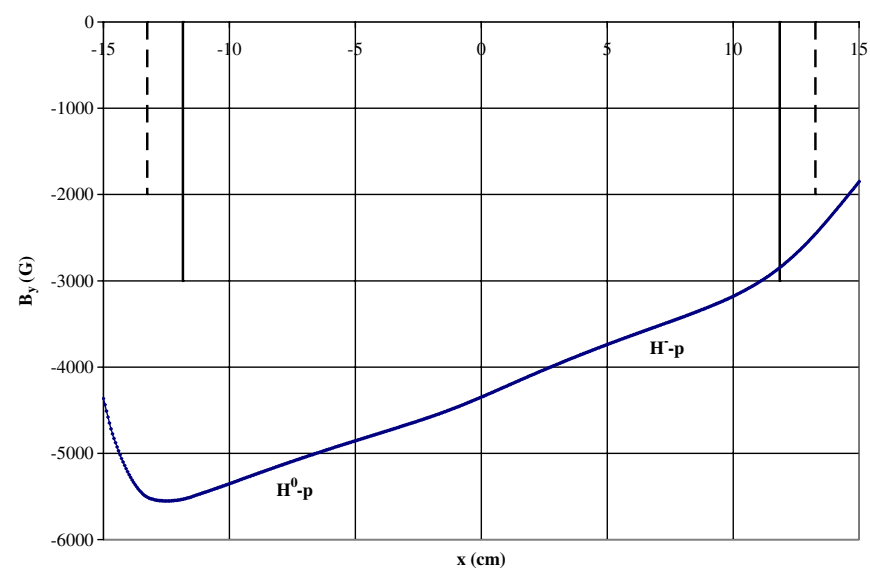

FIG. 4. (Color) Magnetic field on midplane across the IDSM gap at its longitudinal center. 


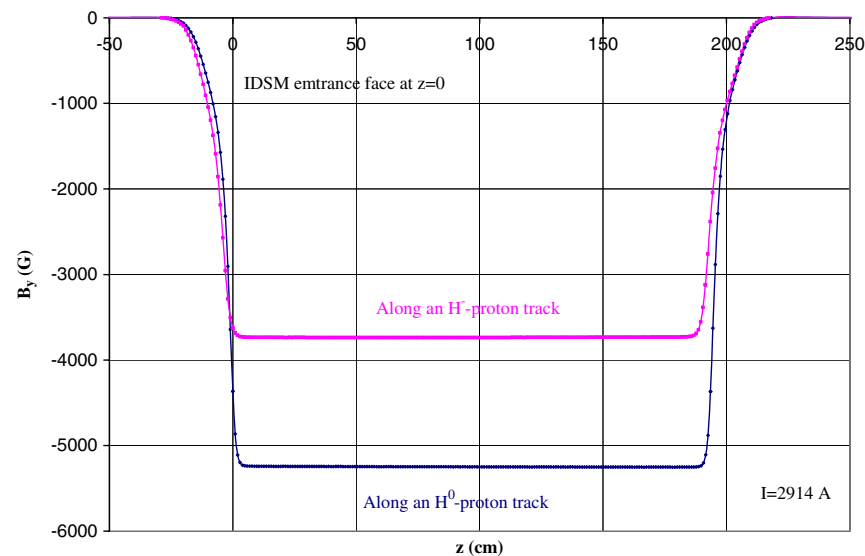

FIG. 5. (Color) Magnetic field along two selected waste beam trajectories on IDSM midplane.

inner surfaces. The $B_{y}$ plot indicates that the dipole has a gradient, which provides horizontal focusing and vertical defocusing for the waste beams. The average slope of the $B_{y}$ curve on the left side of the pole-tip center is about $0.97 \mathrm{~T} / \mathrm{m}$, while it is $1.14 \mathrm{~T} / \mathrm{m}$ on the other side. Thus, the $\mathrm{H}^{-}$-proton beam is focused stronger in the $x$-direction than the $\mathrm{H}^{0}$-proton beam.

The magnetic field component $B_{y}$ along two selected waste beam trajectories on the midplane is illustrated in Fig. 5, where $z=0$ indicates the IDSM entrance face. Both the $\mathrm{H}^{0}$-proton and $\mathrm{H}^{-}$-proton tracks are obtained by launching $1 \mathrm{GeV}$ particles upstream in perpendicular to the IDSM entrance face. The $\mathrm{H}^{0}$-proton starts at $9 \mathrm{~cm}$ from the IDSM axis, while the $\mathrm{H}^{-}$-proton starts at $4 \mathrm{~cm}$ on the other side of the axis. We pick these positions since the chicane dipole settings usually make the two beams asymmetric with respect to the IDSM axis. The total bending field integral along the $\mathrm{H}^{0}$-proton track is $1.034 \mathrm{~T} \mathrm{~m}$ corresponding to a bending angle of $182.8 \mathrm{mrad}$, while it is $0.7898 \mathrm{~T} \mathrm{~m}$, or $139.6 \mathrm{mrad}$, for the $\mathrm{H}^{-}$-proton track. These bending angles are fairly close to the values required by conditions (5a) and (5b). In reality, both waste beams, especially the $\mathrm{H}^{-}$-proton particles, have large incident angles with respect to the IDSM entrance face. Thus, the actual trajectories would deviate appreciably from these selected tracks, and the magnetic fields along real beam trajectories could be quite different from what shown in the figure.

\section{Initial conditions of test particles}

We take a test particle approach in our calculations of the waste beam particle trajectories. The primary foil F1 is nominally $12 \mathrm{~mm}$ wide; it is mounted at an angle of $30^{\circ}$ with respect to the incoming $\mathrm{H}^{-}$beam. Thus, the incoming beam only sees a foil width of $\pm 5.2 \mathrm{~mm}$ as shown in Fig. 6(a), which is the view towards upstream. Measurements show that the incoming $\mathrm{H}^{-}$beam has a slightly elliptical profile with its long axis of about
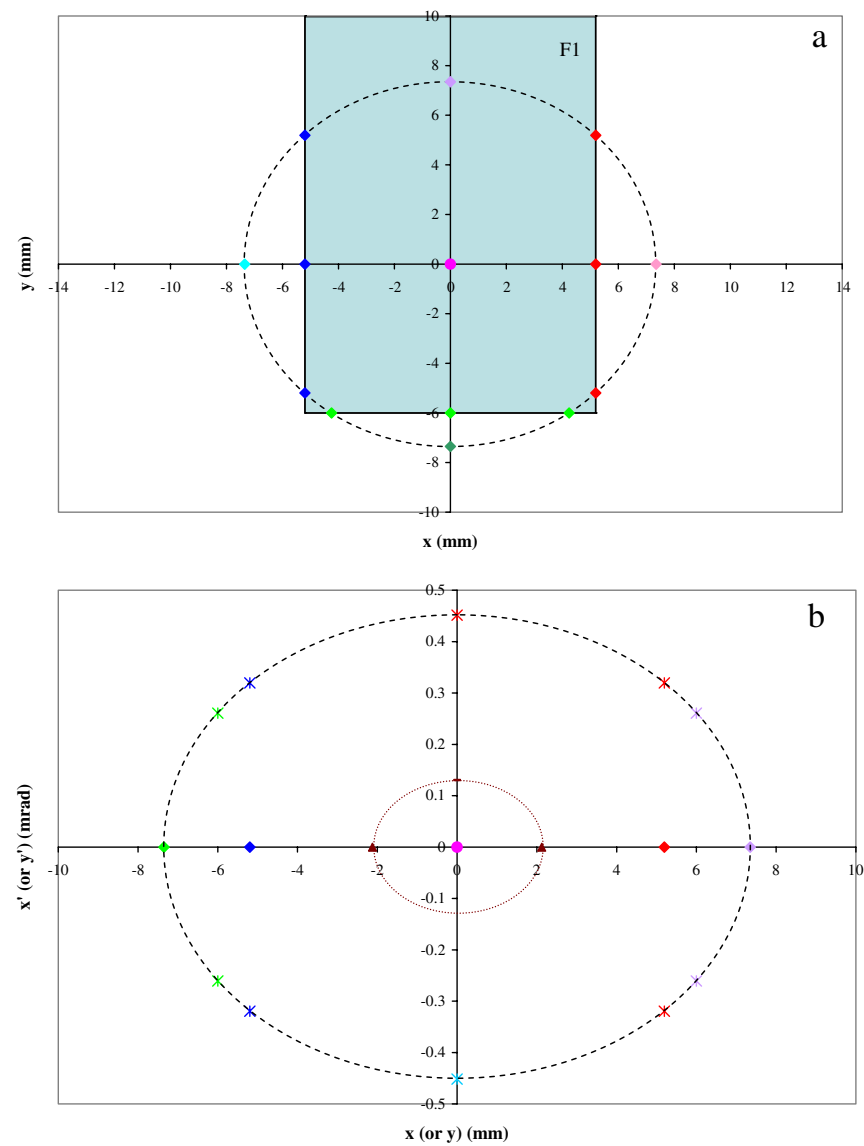

FIG. 6. (Color) (a) Initial test particles in configuration space. (b) Initial test particles in phase space.

$15 \mathrm{~mm}$ in the $y$-direction. We use a round beam profile for the convenience of calculations, and thus we slightly overestimate the waste beam dimension in the $x$-direction. The $\mathrm{H}^{0}$ particles are generated in the foil area, and we pick three of them each on the left (blue diamonds) and right (red diamonds) edges and one each on top (purple diamond) and bottom (green diamond). The majority of the $\mathrm{H}^{-}$particles after $\mathrm{F} 1$ should come from outside the foil, i.e., the three island areas. We pick four of them each on the left (blue diamonds), right (red diamonds), and bottom (green diamonds). Particles with their initial velocity parallel to the $z$-axis are represented by solid diamonds in our plots later. The particles from the F1 center (3.997, 2.3, 30.71 ) are shown by a magenta dot for the $\mathrm{H}^{0}$ centroid and a magenta circle for the $\mathrm{H}^{-}$centroid.

The assignment of initial transverse angles for the test particles is based on the following considerations. The normalized rms emittance of the injected $\mathrm{H}^{-}$beam is $0.5 \pi \mathrm{mm} \mathrm{mrad}$ and the rms radius is about $1.5 \mathrm{~mm}$ [8]. Since the beam has halo tails, its profile is not a true Gaussian, and it occupies much larger area in the real space than expected from a Gaussian distribution. Thus, we employ the concept of equivalent beams [18], which satisfy the Boltzmann-Maxwell distribution in phase 
space. This beam has an effective rms radius of $2.1 \mathrm{~mm}$ and an unnormalized $\mathrm{rms}$ emittance of $0.28 \pi \mathrm{mmmrad}$. Figure 6(b) illustrates two different phase space boundaries for this beam: one-rms (plum dashed ellipse) and 12-times rms emittance (black dashed ellipse). A beam with the Boltzmann-Maxwell distribution in phase space has only $0.25 \%$ particles outside the 12-times rms emittance area. This provides reasonably good criteria for cutoff in calculations. The particles launched at the 12-times rms emittance boundaries with nonzero initial angles are denoted by crosses as shown later in Figs. 10 and 11, etc. The scattering in F1 is negligibly small and is ignored. The scattering in F2 may play some role in spreading particle distributions and causing particle losses, but that effect is not included in these calculations.

\section{3D PARTICLE TRAJECTORIES THROUGH IDSM}

OPERA3D has a built-in TRACK command for calculating trajectories of charged particles by directly integrating the equations of motion in simulated magnetic fields. We launch waste beam particles of $1 \mathrm{GeV}$ in energy at F1 with different initial locations and slopes as specified in Figs. 6(a) and 6(b). The $\mathrm{H}^{0}$ particles do not feel any force in between F1 and F2. They simply drift in the region and the calculation of their trajectories actually starts at F2. For the $\mathrm{H}^{-}$particles, we first compute their trajectories in between $\mathrm{F} 1$ and F2, and obtain their positions and velocities at F2. This information is used as the initial conditions of the waste $\mathrm{H}^{-}$-proton beam at $\mathrm{F} 2$. The new Euler's angles at F2 are $\mathrm{phi}=\operatorname{Atan}\left(v_{y} / v_{x}\right)$, theta $=\operatorname{Atan}\left(v_{\perp} / v_{z}\right)$, and $\mathrm{psi}=$ 0 , where $v_{\perp}$ is the transverse velocity of the $\mathrm{H}^{-}$particles arriving at $\mathrm{F} 2$.

Figure 7 shows the $\mathrm{H}^{-}$particle trajectories (in between $\mathrm{F} 1$ and $\mathrm{F} 2$ ) and $\mathrm{H}^{-}$-proton particle trajectories (after F2) in the $y-z$ plane. Three chicane dipoles D2, D3, and D4 in the production setting are depicted by their axial positions, and IDSM is indicated by the inner surface of its vacuum chamber. The simulation model extends only to $z=$ $1000 \mathrm{~cm}$, beyond which the particle trajectories are obtained by linear extrapolation. The trajectory plot stops at a $z$-position marked by a light blue cross, which is $1.3 \mathrm{~m}$ from the downstream quadrupole magnet center. It is clear that all the $\mathrm{H}^{-}$-proton particles have significant $y$-motion in D4 and IDSM, and some $\mathrm{H}^{-}$-proton particles are lost to the upper surface of the IDSM vacuum chamber. These losses are much more serious in the design setting and the delivered setting of the chicane dipoles.

Figure 8 shows the $\mathrm{H}^{0}$ particle trajectories (in between $\mathrm{F} 1$ and $\mathrm{F} 2$ ) and $\mathrm{H}^{0}$-proton particle trajectories (after F2) in the $y$ - $z$ plane. In comparison with Fig. 7, the $y$-motion of all the $\mathrm{H}^{0}$-proton particles in the D4 region is unnoticeable, and is still quite moderate in the second half of IDSM. We do not see any losses of these particles in the $y$-direction. This is also true for the other two settings.

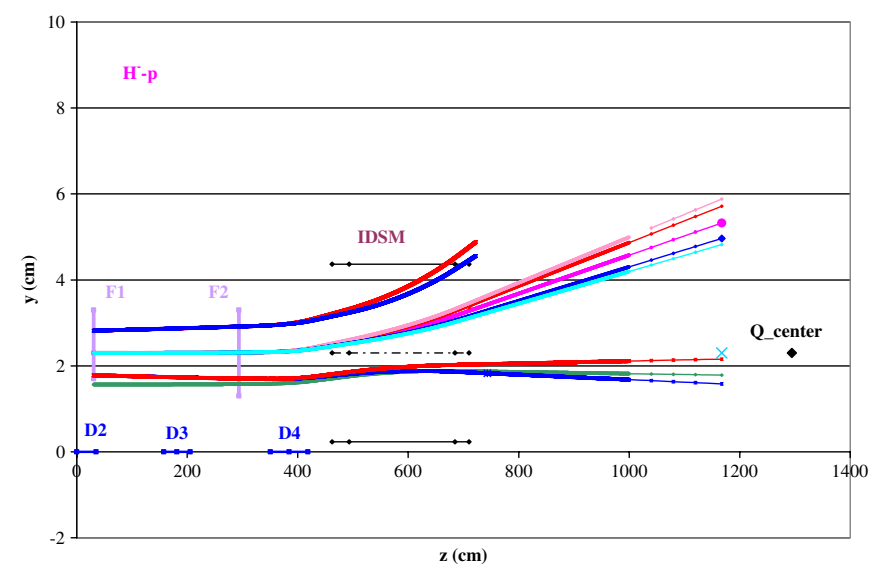

FIG. 7. (Color) $\mathrm{H}^{-}$and $\mathrm{H}^{-}$-proton particle trajectories in the $y-z$ plane.

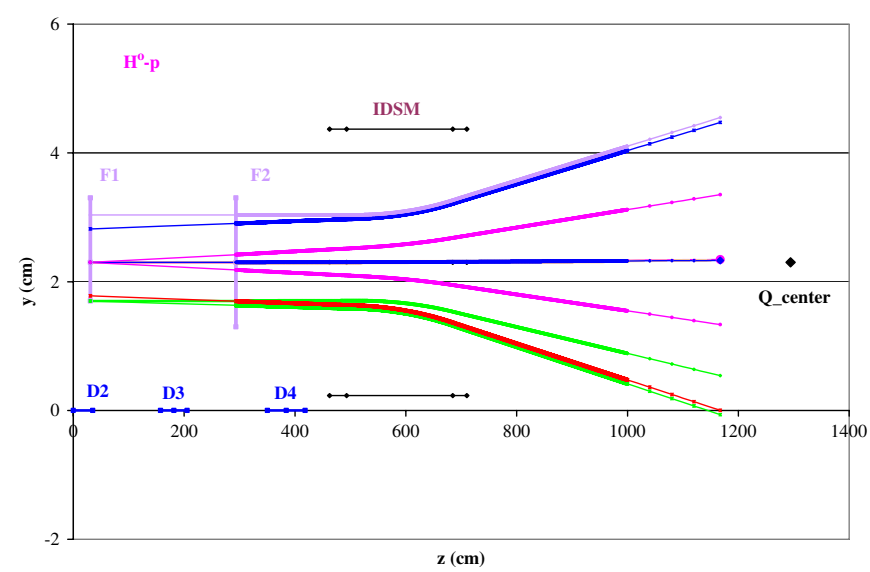

FIG. 8. (Color) $\mathrm{H}^{0}$ and $\mathrm{H}^{0}$-proton particle trajectories in the $y-z$ plane.

Figure 9 shows the waste beam trajectories in the $x-z$ plane. The two bundles of the $\mathrm{H}^{-}$-proton and $\mathrm{H}^{0}$-proton beams include their centroids and two more tracks in the maximum $x$-extension. We can see that the $\mathrm{H}^{0}$-proton beam is too close to the IDSM vacuum chamber wall,

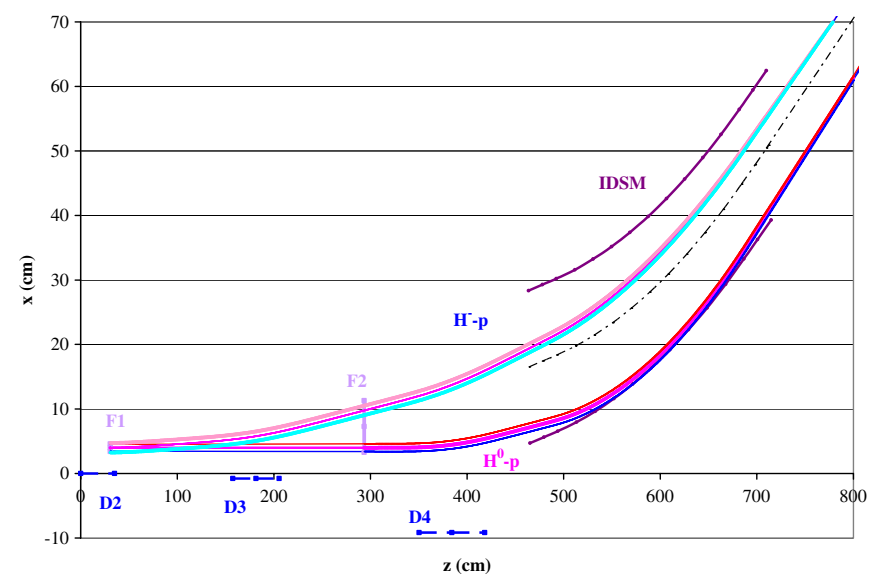

FIG. 9. (Color) Waste beam trajectories in the $x-z$ plane. 
and some particles already hit the middle of the IDSM vacuum chamber. This is consistent with what is observed in operation. These horizontal losses for the $\mathrm{H}^{0}$-proton particles are not seen in the two other settings since they have stronger bending angles in D4. This will be explained in more detail in Sec. VIB.

The waste beam trajectories in IDSM shown in Fig. 9 are quite different from the selected tracks used for the $B_{y}$ plot in Fig. 5, since their initial conditions before the IDSM entrance are quite different. The calculations show that in the IDSM coordinates, where the origin is at the center of the IDSM pole-tip entrance face, the $\mathrm{H}^{0}$-proton centroid in Fig. 9 arrives in front of the septum at $x=-10.9$, $y=0.0033$, and $z=-30.7 \mathrm{~cm}$ with the incident angles of $\operatorname{Atan}\left(v_{x} / v_{z}\right)=-6.5 \mathrm{mrad}$ and $\operatorname{Atan}\left(v_{y} / v_{z}\right)=$ $0.022 \mathrm{mrad}$. The $\mathrm{H}^{0}$-proton centroid is too far away from the IDSM longitudinal axis; and the negative incident angle of $-6.5 \mathrm{mrad}$ in the horizontal direction drives it further toward the vacuum chamber wall. Note that the vacuum chamber has a horizontal aperture of only $\pm 11.85 \mathrm{~cm}$. Thus, it is easy to understand that some $\mathrm{H}^{0}$-proton particles around their centroid would hit the vacuum chamber wall somewhere inside IDSM. On the other hand, the $\mathrm{H}^{-}$-proton centroid arrives in front of IDSM at $x=1.29, y=0.186$, and $z=-30.4 \mathrm{~cm}$ with the incident angles of $\operatorname{Atan}\left(v_{x} / v_{z}\right)=30.1 \mathrm{mrad}$ and $\operatorname{Atan}\left(v_{y} / v_{z}\right)=2.3 \mathrm{mrad}$. Though the $\mathrm{H}^{-}$-proton centroid is initially very close to the IDSM pole-tip center, its large incident angle of $30.1 \mathrm{mrad}$ causes it to gradually deviate more and more from the IDSM axis. This mismatch between the IDSM design parameters and initial conditions makes it very difficult to transport the two waste beams through IDSM, as well as the rest of the way to the dump.

Figure 10 shows all the waste beam particles at the IDSM entrance looking upstream. The blue dashed line indicates the IDSM midplane position. The pattern of the $\mathrm{H}^{0}$-proton test particles is very similar to that at $\mathrm{F} 1$. But, the $\mathrm{H}^{-}$-proton particles already gain significant $y$-motion, and their centroid is $\Delta y=0.26 \mathrm{~cm}$ above the IDSM mid-

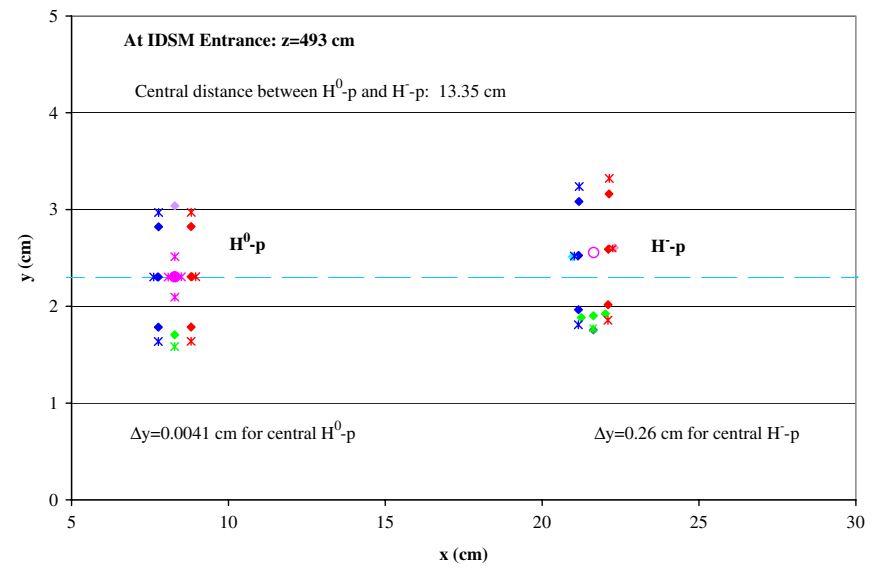

FIG. 10. (Color) Waste beam particles at the IDSM entrance.

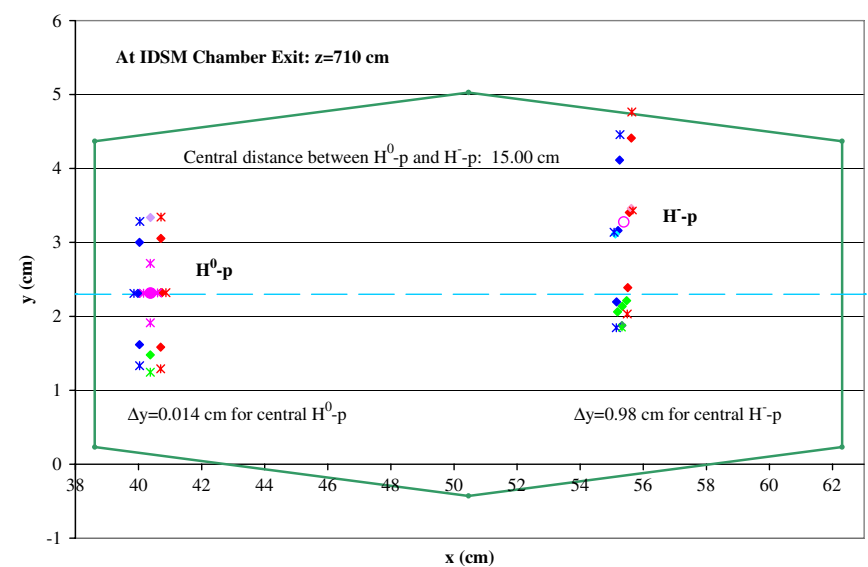

FIG. 11. (Color) Waste beam particles at the IDSM chamber exit.

plane. The distance between the $\mathrm{H}^{0}$-proton centroid and the $\mathrm{H}^{-}$-proton centroid is about $13 \mathrm{~cm}$. The focusing of the $\mathrm{H}^{-}$-proton particles in the $x$-direction and defocusing in the $y$-direction can also be seen. This is due to the edge focusing in the D4 fringe field.

Figure 11 shows all the waste beam particles at the IDSM chamber exit, again looking upstream. The $\mathrm{H}^{-}$-proton particles centroid is $\Delta y=0.98 \mathrm{~cm}$ above the midplane and some of them are already outside the vacuum chamber. The horizontal distance between the $\mathrm{H}^{0}$-proton centroid and the $\mathrm{H}^{-}$-proton centroid is increased to about $15 \mathrm{~cm}$. But, the $\mathrm{H}^{0}$-proton particles are very close to the chamber wall in the horizontal direction. This is consistent with the plot in Fig. 9. The vertical separation and horizontal distance between the two waste beam centroids are important parameters in beam measurements as explained in Sec. VID. In Fig. 11, we can also see that the $\mathrm{H}^{-}$-proton particles are much more strongly focused in the $x$-direction and defocused in the $y$-direction than the $\mathrm{H}^{0}$-proton particles. This is not only due to the higher gradient term inside IDSM for the $\mathrm{H}^{-}$-proton particles (Fig. 4), but also due to much stronger edge focusing/defocusing of the $\mathrm{H}^{-}$-proton particles, which are incident to IDSM with large facerotation angles.

In the delivered setting and the design setting, there are even more $\mathrm{H}^{-}$-proton particles outside the vacuum chamber in Fig. 11. More $\mathrm{H}^{-}$-proton particles are lost on the upper surface of the septum vacuum chamber. On the other hand, the $\mathrm{H}^{0}$-proton particles in these two settings have good distances from the vacuum chamber wall in the horizontal direction, and for these cases the $\mathrm{H}^{0}$-proton particle losses in the septum may not be a problem.

\section{PARTICLE OPTICS THROUGH QUADRUPOLE MAGNET TO DUMP}

The waste beam transport after IDSM through the quadrupole magnet to the injection dump is shown in Fig. 12. The region from $z=-1.3$ to $z=1.3 \mathrm{~m}$ is for the quad- 


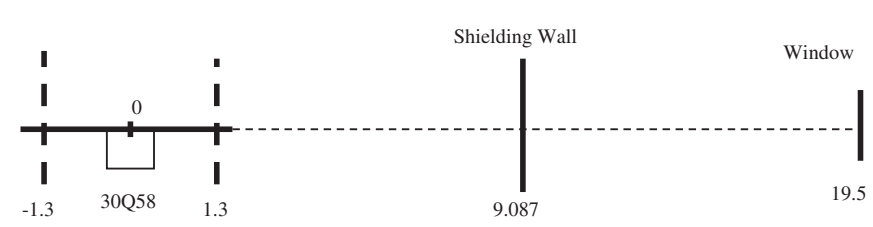

FIG. 12. Waste beam transport through a quadrupole magnet to dump (dimension in meters).

rupole magnet $30 \mathrm{Q} 58$, including its fringe field. The remaining is just a drift space with the shielding wall at $z=$ $9.087 \mathrm{~m}$ and the injection dump window at $19.5 \mathrm{~m}$. For the particle optics analyses, we first transform the information in the previous section to the quadrupole magnet frame to obtain the initial conditions at $z=-1.3 \mathrm{~m}$. Figures 13(a) and 13(b) show the waste beam phase space distributions in the $x-x^{\prime}$ and $y-y^{\prime}$ phase spaces. It is easy to see that the waste beam is converging in $x$ and diverging in $y$, requiring a horizontally defocusing quadrupole magnet.

The quadrupole magnet 30Q58 was originally designed for the ring straight sections, and its performance charac-
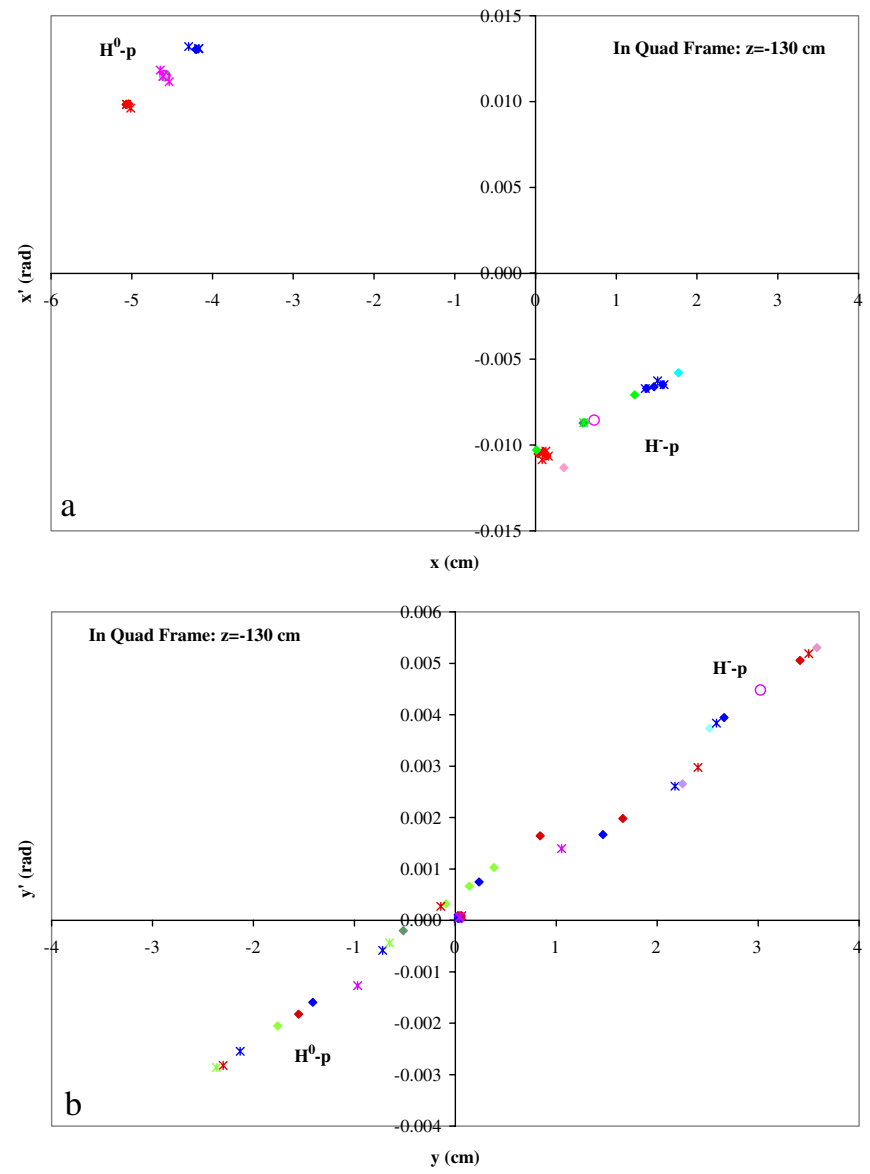

FIG. 13. (Color) (a) Phase space distribution of waste beams in $x-x^{\prime}$ space at the quadrupole magnet entrance. (b) Phase space distribution of waste beams in $y-y^{\prime}$ space at the quadrupole magnet entrance, where the vacuum chamber aperture is $9.86 \mathrm{~cm}$.

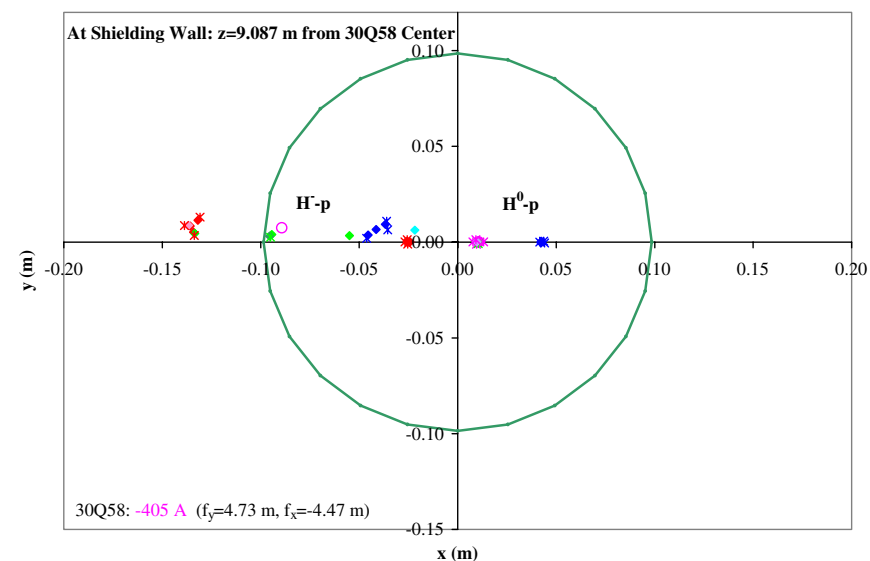

FIG. 14. (Color) Waste beam particle positions at shielding wall for the quadrupole current of $-405 \mathrm{~A}$.

TABLE II. $30 \mathrm{Q} 58$ parameters at $I=405$ A.

\begin{tabular}{lccrcr}
\hline \hline & $m_{11}$ & $m_{12}(\mathrm{~m})$ & \multicolumn{1}{c}{$m_{21}(1 / \mathrm{m})$} & $m_{22}$ & \multicolumn{1}{c}{$f(\mathrm{~m})$} \\
\hline $\mathrm{F}$ & 0.7240 & 2.2508 & -0.2114 & 0.7240 & 4.731 \\
$\mathrm{D}$ & 1.2893 & 2.9638 & 0.2235 & 1.2893 & -4.475 \\
\hline \hline
\end{tabular}

teristics have been well studied [19,20]. Instead of a simple hard edge model for the quadrupole magnet, we employ its transfer matrices rigorously computed from the equations of motion with the fringe field effect [21]. We first use the transfer matrices to map the waste beam particles from $z=$ -1.3 to $z=1.3 \mathrm{~m}$, and then to use the drift space matrices to map the particles further downstream. A number of different quadrupole magnet currents are used in calculations and the best result is shown in Fig. 14, where the quadrupole magnet parameters are listed in Table II, and the green circle indicates the inner surface of the vacuum chamber. A single quadrupole magnet $30 \mathrm{Q} 58$ cannot transport all the waste beam particles even through the shielding wall, let alone the dump window which is still more than ten meters downstream. For the design setting and the delivered setting, the situations are even worse.

The injection dump optics in the original design $[7,22]$ shows that the beta function for both $x$ and $y$ at the dump window reaches approximately $3500 \mathrm{~m}$. With a $10^{\prime \prime}$ pipe for the transport channel, the acceptance is about $4.4 \pi \mathrm{mm} \mathrm{mrad}$. Though this acceptance is significantly larger than the rms emittance of the linac injected $\mathrm{H}^{-}$ beam, it is far less than the equivalent phase space area occupied by both $\mathrm{H}^{0}$-proton and $\mathrm{H}^{-}$-proton waste beam particles, as shown in Figs. 13(a) and 13(b). This makes the waste beam transport downstream to the dump window impossible.

\section{REMEDIES}

The studies of 3D particle trajectories in Sec. IV and particle optics in Sec. V have shown three waste beam loss 
mechanisms. First, the $\mathrm{H}^{-}$-proton particle motion in the $y$-direction is excessive and the vertical aperture of the IDSM vacuum chamber is too small. Many $\mathrm{H}^{-}$-proton particles are lost on the upper surface of IDSM. This is true for all the three sets of chicane dipole settings. Second, many $\mathrm{H}^{0}$-proton particles are lost in the horizontal direction in the middle of IDSM for the production setting. For the other two settings, these losses do not appear in the simulations since they employ stronger bending field in D4. Third, by employing only a single quadrupole magnet, the waste beams cannot be transported through the shielding wall in any of the three chicane dipole settings. After more detailed investigations of the three beam loss mechanisms, we have devised solutions to the problems as described below.

\section{A. $\mathrm{H}^{-}$-proton particle losses in the $\boldsymbol{y}$-direction in IDSM}

The $y$-motion of the $\mathrm{H}^{-}$-proton particles in the chicane region is mainly caused by $\mathrm{D} 4$, as shown for the production setting in Fig. 15. This is because the $\mathrm{H}^{-}$-proton trajectories pass through the D4 pole-tip boundary, where the magnetic field is very nonuniform and there is a significant $B_{x}$ component. Figure 16 shows the geometry in the D4 region, and Fig. 17 plots the magnetic field along the $\mathrm{H}^{-}$-proton centroid trajectory through $\mathrm{D} 4$. The integrated $B_{x}$ is about $6 \%$ of the $B_{y}$ integral for the production setting. The ratio of the $B_{x}$ integral over the $B_{y}$ integral through D4 is more than $10 \%$ with the delivered setting. If we slide D4 in the positive $x$-direction, the $\mathrm{H}^{-}$-proton tracks would move towards the D4 center into a more uniform field region. The $B_{x}$ component is then much reduced, and the $y$-motion of the $\mathrm{H}^{-}$-proton particles is greatly mitigated. The minimum movement is $\Delta x=8 \mathrm{~cm}$ due to mechanical constraints.

We have built new simulation models, in which the D4 center is moved from the original $x=-9.155$ to $x=$ $-1.155 \mathrm{~cm}(\Delta x=8 \mathrm{~cm})$. Figure 18 shows the $y$-motion

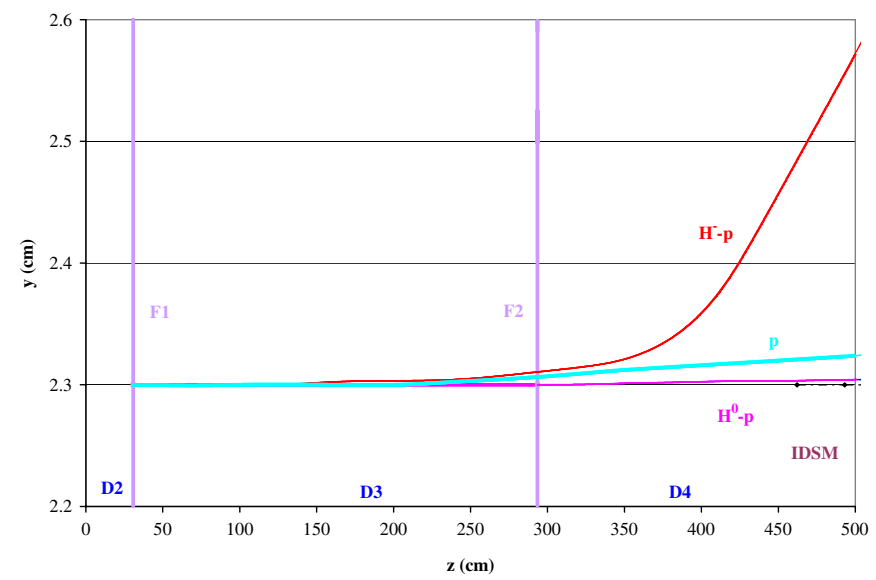

FIG. 15. (Color) Waste beam particle trajectories in the $y-z$ plane in the region of chicane dipoles. of the $\mathrm{H}^{-}$-proton particles in the new configuration under the production setting. All the particles remain inside IDSM, in contrast to that in Fig. 7. This has been verified to also be true for the other two settings. Thus, moving D4

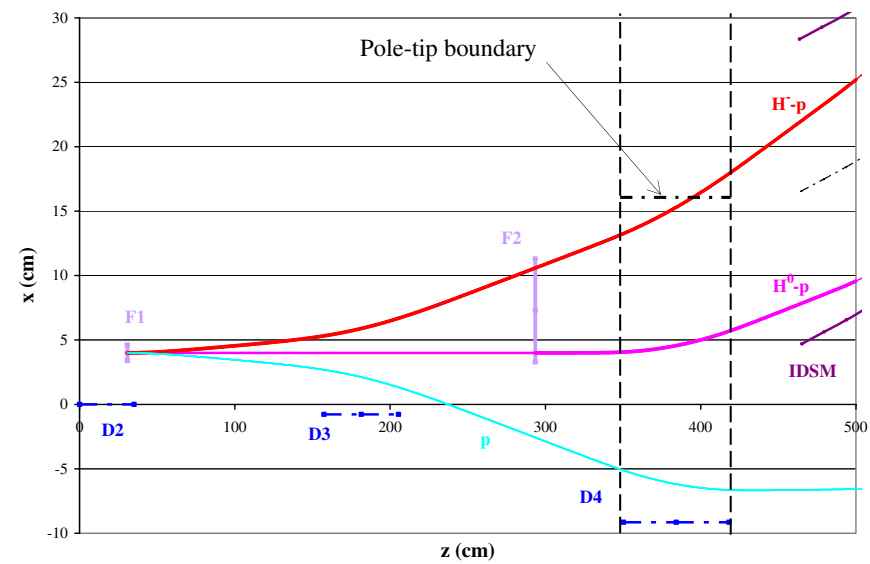

FIG. 16. (Color) Particle tracks in the D4 region and vicinity in the $x-z$ plane, where the D4 axis is at $x=-9.155 \mathrm{~cm}$.

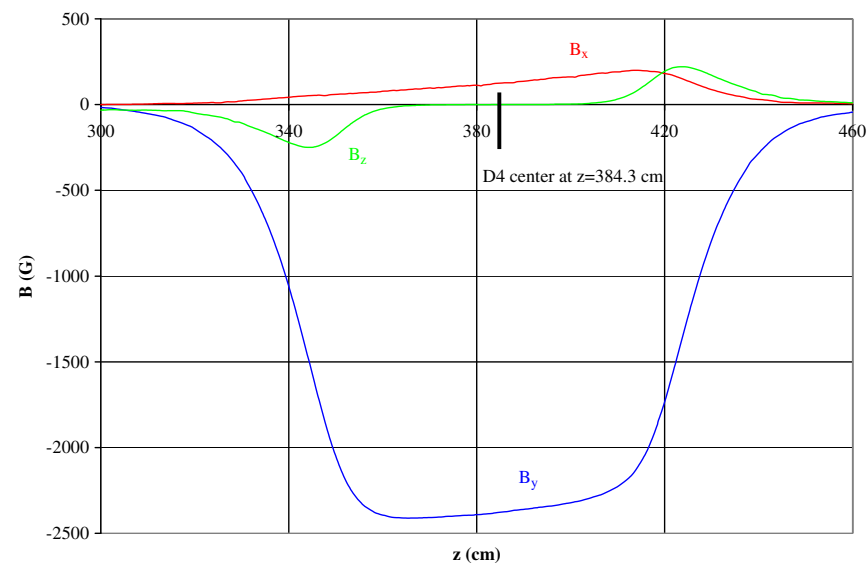

FIG. 17. (Color) Field distribution along the $\mathrm{H}^{-}$-proton centroid track in the $\mathrm{D} 4$ region.

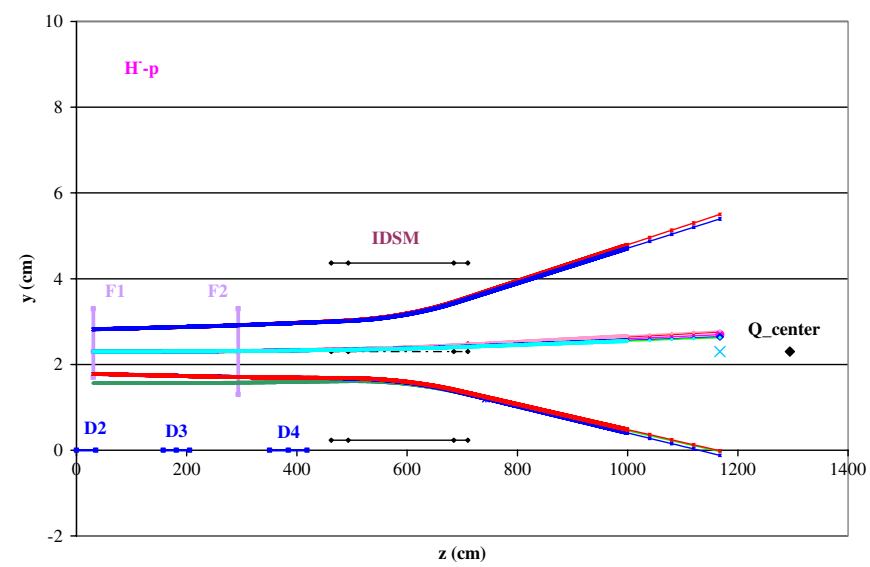

FIG. 18. (Color) The $\mathrm{H}^{-}$-proton particle trajectories in the $y-z$ plane after the D4 move by $\Delta x=8 \mathrm{~cm}$. 
by $\Delta x=8 \mathrm{~cm}$ would significantly reduce the $y$-motion of the $\mathrm{H}^{-}$-proton particles, as well as their losses. On the other hand, the move would push the circulating proton beam towards the nonuniform field region in D4. This has been checked in a separate simulation, which shows unnoticeable effect on the circulating proton beam after D4 is moved by $\Delta x=8 \mathrm{~cm} \mathrm{[23].}$

\section{B. $\mathbf{H}^{\mathbf{0}}$-proton particle losses in the $\boldsymbol{x}$-direction in IDSM}

The waste beam trajectories in IDSM are very sensitive to the chicane dipole settings. With the production setting, where the D4 field strength is smaller than in the other two settings, many $\mathrm{H}^{0}$-proton particles would be lost in the horizontal direction around the middle of IDSM. Since the IDSM cannot be moved in the minus $x$-direction, a possible remedy for this problem is to move the injection point at $\mathrm{F} 1$ in the positive $x$-direction. This leads to the $\mathrm{H}^{0}$-proton tracks off the IDSM vacuum chamber wall. Figure 19 shows the waste beam particle trajectories in the $x$-z plane for the production setting model with F1 moved by $\Delta x=$ $1 \mathrm{~cm}$ and injection at $1 \mathrm{mrad}$ angle with respect to the $z$-axis. It appears that this is a reasonable solution. This also requires an adjustment of the dynamic bump amplitude by $+1 \mathrm{~cm}$ to keep the painting parameters unchanged. Moreover, the F1 longitudinal position should be slightly ahead of the static bump peak in order to accommodate the $1 \mathrm{mrad}$ injection angle for good injection.

An alternative way to accomplish the same task is to develop a new chicane dipole setting. To accommodate the F1 move by $\Delta x=1 \mathrm{~cm}$, we can increase the static bump height to $10.66 \mathrm{~cm}$ from the original $9.66 \mathrm{~cm}$ as calculated in Sec. II A. The primary foil F1 will stay at the peak of the static bump. In order to inject at right position with zero angle with respect to the $z$-axis, the incoming $\mathrm{H}^{-}$beam line has to be adjusted as well to be parallel with the $z$-axis at F1. This may not be a trivial task. From Eqs. (1)-(4), we obtain the following bending angles: 46.35, -58.60, -31.19 , and $43.44 \mathrm{mrad}$ for chicane dipoles D1, D2, D3,

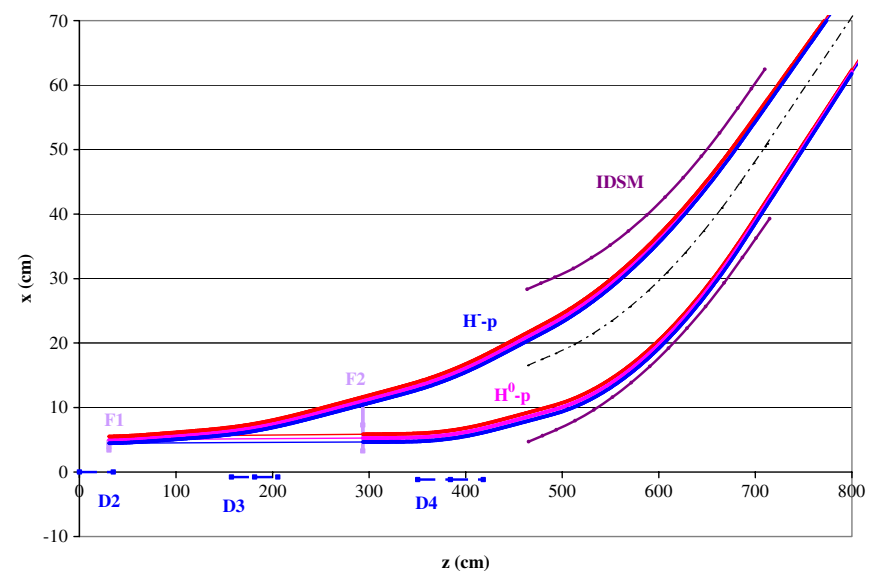

FIG. 19. (Color) Waste beam trajectories in the $x-z$ plane after $\mathrm{F} 1$ move by $\Delta x=1 \mathrm{~cm}$ and with injection at $1 \mathrm{mrad}$. and D4, respectively. This setting provides good injection and guarantees a closed injection bump and a good equilibrium orbit for the circulating proton beam. In addition, the new D4 bending angle (43.44 mrad) is larger than that in the production setting (39.4 mrad). This is good for the $\mathrm{H}^{0}$-proton beam in terms of its clearance from the IDSM chamber wall in the horizontal direction.

We have built a 3D simulation model under the new chicane dipole setting with the $\mathrm{D} 4$ position already moved by $\Delta x=8 \mathrm{~cm}$. The calculations have been done with the initial centroid particles at $x=4.997 \mathrm{~cm}$, i.e. $\Delta x=1 \mathrm{~cm}$ from that in the original production setting. Figure 20 shows the waste beam particle trajectories in the $x-z$ plane. The $\mathrm{H}^{0}$-proton particles are kept clear of the IDSM vacuum chamber wall. In comparison with Fig. 9 for the production setting, this is a much better transport for the waste beam particles through IDSM. In the new chicane dipole setting with $\mathrm{D} 4$ moved by $\Delta x=8 \mathrm{~cm}$, both the $\mathrm{H}^{0}$-proton beam and the $\mathrm{H}^{-}$-proton beam go through IDSM without showing any losses in the $y$-direction. Figure 21 shows all the

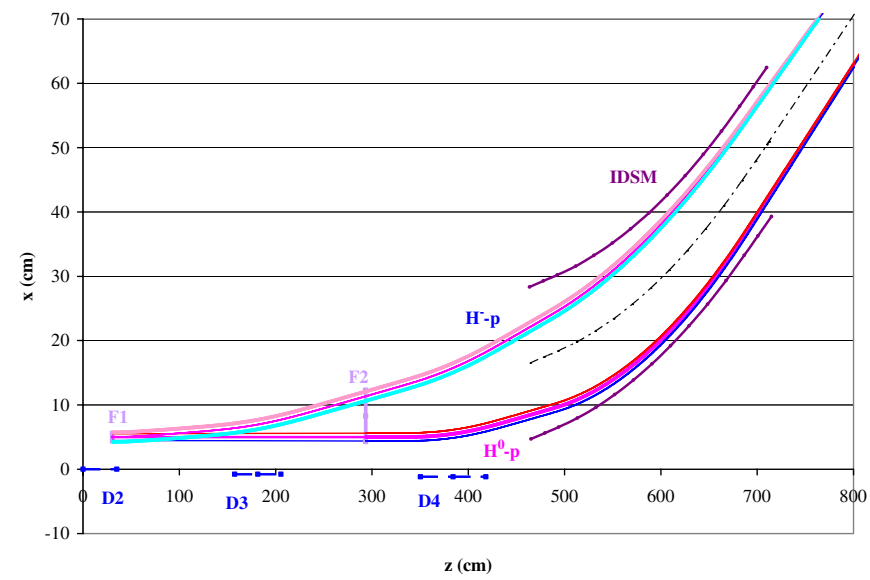

FIG. 20. (Color) Waste beam trajectories in the $x$-z plane under the new chicane dipole setting.

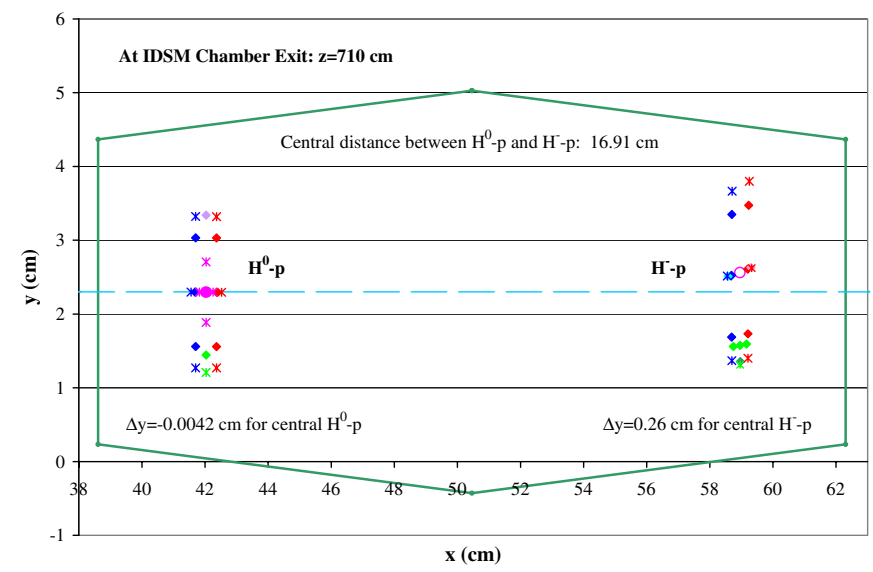

FIG. 21. (Color) All waste beam particles at the IDSM chamber exit in the new chicane dipole setting. 
waste beam particles at the IDSM chamber exit. The $\mathrm{H}^{-}$-proton centroid is $\Delta y=0.26 \mathrm{~cm}$ above the middle plane, as indicated by the dashed blue line, and it is about $16.9 \mathrm{~cm}$ from the $\mathrm{H}^{0}$-proton centroid. The two bunches of the waste beam particles are symmetrically distributed in the horizontal direction with respect to the IDSM center.

We are also modifying a spare IDSM for the improvement of its performance. First, the vertical gap will be increased by $2 \mathrm{~cm}$, which will provide the $\mathrm{H}^{-}$-proton particles more clearance in the $y$-direction. Second, good field region for the $\mathrm{H}^{0}$-protons will be further extended from the pole-tip edge toward the septum plate, where it happens that there is some space available. Thus, the vacuum chamber can be accordingly enlarged by $5 \mathrm{~cm}$, which will give the $\mathrm{H}^{0}$-proton particles significantly more clearance in the horizontal direction. Third, we will add specially designed $z$-bumps on the IDSM entrance face for the $\mathrm{H}^{0}$-proton beam that will further push these particles inward in IDSM, and reduce the distance between the $\mathrm{H}^{0}$-proton centroid and the $\mathrm{H}^{-}$-proton centroid at the IDSM exit. We hope all of these efforts will reduce the waste beam losses in IDSM to the lowest level.

\section{Waste beam losses downstream after quadrupole magnet}

The easiest and most effective way to solve the transport problem after the quadrupole magnet (30Q58) is to add another quadrupole magnet such as a $30 \mathrm{Q} 44$ to form a quadrupole doublet. The doublet can reduce the beta function amplitude downstream and make the beam transport there possible. This approach is rather straightforward, and we skip the details. We are now investigating the possibility of adding a second quadrupole magnet after 30Q58 in the near future.

\section{Experimental verifications}

Beam measurements have been made to compare with model predictions. Here we report some results before and after the D4 move. The comparison between early measurements and simulations is qualitative, while recent measurements are more quantitative.

Before we produced preliminary results in 3D simulation studies, many beam measurements during the commissioning and early operation were already performed. Measurements of the waste beam parameters indicated qualitative agreement with the simulation models. For example, since the SNS ring commissioning the particle losses along the entire injection dump line were very high, especially at IDSM. Activation measurements showed elevated levels about halfway along the length of IDSM on the beam right side (looking downstream), indicating that the $\mathrm{H}^{0}$-proton waste beam was in fact scraping on the IDSM vacuum chamber. This was in good agreement with the trajectories shown in Fig. 9. Another example was the vertical motion of the $\mathrm{H}^{-}$-proton beam in experiments.
Beam position measurements showed a 42 to $47 \mathrm{~mm}$ vertical separation of the $\mathrm{H}^{0}$-proton beam and the $\mathrm{H}^{-}$-proton beam at a beam position monitor BPM01 located immediately downstream of the 30Q58 quadrupole magnet. The simulation models with three different chicane dipole settings, i.e., the production, delivered, and design settings, yielded a vertical separation of the two waste beam centroids at the same location in the range of 30 to $73 \mathrm{~mm}$. Both the measurements and simulations resulted in the same order of magnitude for the $\mathrm{H}^{-}$-proton vertical motion at BPM01. No further simulation effort was made to better compare the BPM01 signal at the exact experimental conditions, such as beam energy, injection parameters, etc., in addition to the chicane dipole settings.

More quantitative comparison between measurements and simulations has been made recently after chicane dipole D4 was moved by $\Delta x=8 \mathrm{~cm}$. New diagnostics such as a wire scanner WSO0 and a BPM00 have also been installed just after IDSM. The coordinates of WS00 and BPM00 in the models are $(78.15,2.3,834.11)$ and (106.53, 2.3, 961.44). Figure 22 shows the measurement from WS00, where the two waste beams are separated by $149 \mathrm{~mm}$ in the horizontal plane. A new simulation model with the same chicane setting, same beam energy, and same injection conditions has been built. The model yields a horizontal separation of $136 \mathrm{~mm}$ for the two waste beam centroids at the WS00 location. The discrepancy is about 9\%. Considering all the errors in the experimental setup and the diagnostic parameters, the agreement between the WS00 measurement and the new simulation result is quite good. The new model also predicts a vertical separation of $2.4 \mathrm{~mm}$ for the two waste beam centroids at the WSO0 location. This is too small to be accurately measured by WS00. As an independent verification of the vertical motion, measurements at BPM00 show an $\mathrm{H}^{0}$-proton beam and $\mathrm{H}^{-}$-proton beam vertical separation of $4.5 \mathrm{~mm}$. The new simulation model yields $3.2 \mathrm{~mm}$ for this separation at

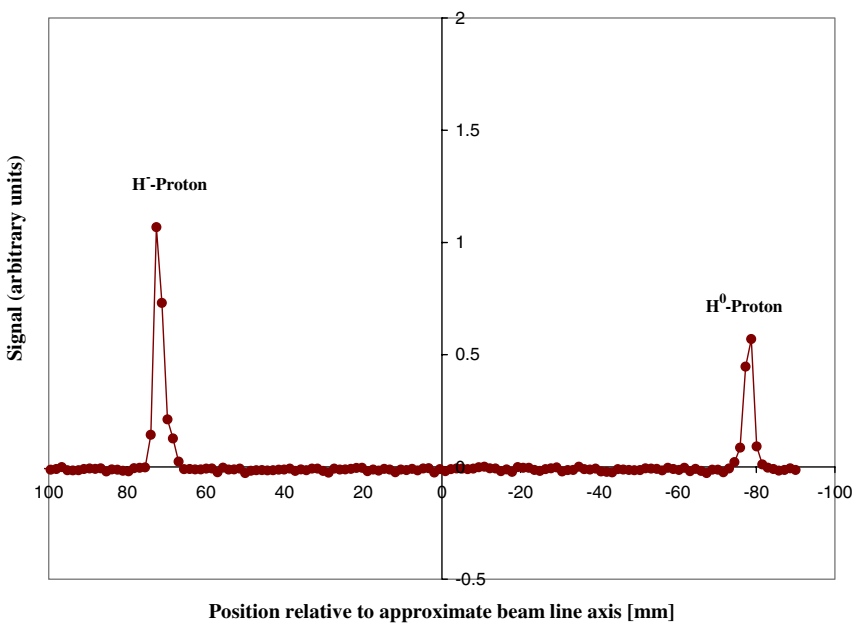

FIG. 22. (Color) Horizontal separation of two waste beams measured by WS00. 
the BPM00 location, again in good agreement with the measurement considering the 1-2 $\mathrm{mm}$ measurement error. In comparison with the situation before the D4 move, the $y$-motion of the $\mathrm{H}^{-}$-proton beam has been dramatically reduced by 1 order of magnitude. Indeed, this problem no longer exits for the SNS ring injection operation.

\section{SUMMARY}

Three-dimensional simulation models of the SNS ring injection dump beam line have been built to study the waste particle trajectories and beam losses. The models consist of three chicane dipoles and one injection dump septum magnet. The waste beam particle trajectories are obtained in the models with various chicane dipole settings. The particle optics downstream of the septum is also analyzed for various situations.

The studies have clearly demonstrated three beam loss mechanisms in the injection dump line design and operation. First, the vertical motion of the waste beams plays an important role in beam dynamics. Many $\mathrm{H}^{-}$-proton particles are lost on the upper surface of the IDSM vacuum chamber. Second, the horizontal motion of the waste beam particles is very sensitive to the chicane dipole settings. Many $\mathrm{H}^{0}$-proton particles are lost to the IDSM vacuum chamber in the horizontal direction when the production setting of the chicane dipoles is employed. This is caused by inadequate bending of chicane dipole D4. Third, a single quadrupole magnet downstream is practically not possible to transport all the waste beam particles to the injection dump window. This is because the equivalent phase space area occupied by the two waste beams is very large and the acceptance of the channel is not adequate.

The 3D modeling of the injection waste beam dump line has also provided remedies for the beam loss problems. We have found that a move of D4 by $\Delta x=8 \mathrm{~cm}$ can significantly decrease the vertical motion of the $\mathrm{H}^{-}$-protons, and therefore reduce their losses. This has already been implemented and the $\mathrm{H}^{-}$-proton losses in IDSM appear to be much reduced. Beam measurements before and after the D4 move show quite good agreement with the model predictions. We are now working on adjusting the chicane dipole settings to increase the D4 bending angle and to reduce the $\mathrm{H}^{0}$-proton horizontal losses in IDSM. Modification of a spare septum for more aperture and better performance is in progress. Adding a second quadrupole magnet downstream has also been in consideration.

\section{ACKNOWLEDGMENTS}

The authors would like to thank J. Error, J. Galambos, S. Henderson, M. Holding, T. Hunter, B. Kelmers, and G. Murdoch for their help and support in this work. Many interesting discussions on the topic with J. Holmes and Y. Zhang are acknowledged. The help from J. Simkin of VF, England, in OPERA/TOSCA software is greatly appreciated.

[1] R.L. Martin, High-Brightness Beams for Advanced Accelerator Applications, AIP Conf. Proc. No. 253 (AIP, New York, 1991), p. 232.

[2] I. Hofmann, High-Brightness Beams for Advanced Accelerator Applications, AIP Conf. Proc. No. 253 (AIP, New York, 1991), p. 149.

[3] J. Wei, D. T. Abell, J. Beebe-Wang, M. Blaskiewicz, P. R. Cameron, N. Catalan-Lasheras, G. Danby, A. V. Fedotov, C. Gardner, J. Jackson, Y. Y. Lee, H. Ludewig, N. Malitsky, W. Meng, Y. Papaphilippou, D. Raparia, N. Tsoupas, W. T. Weng, R. L. Witkover, and S. Y. Zhang, Phys. Rev. ST Accel. Beams 3, 080101 (2000), http:// prst-ab.aps.org/abstract/PRSTAB/v3/i8/e080101.

[4] J. Wei, in BNL/SNS Technical Note 76, 2000.

[5] J. Wei, J. Beebe-Wang, M. Blaskiewicz, J. Brodowski, A. Fedotov, C. Gardner, Y. Y. Lee, D. Raparia, V. Danilov, J. Holmes, C. Prior, G. Rees, and S. Machida, in Proceedings of the 2001 Particle Accelerator Conference, Chicago, IL, p. 2560.

[6] D. T. Abell, Y. Y. Lee, and W. Meng, in Proceedings of the European Particle Accelerator Conference, Vienna, Austria, 2000, p. 2107.

[7] D. Raparia, A. Jain, W. Meng, Y. Y. Lee, and J. Jackson, in ASAC (Accelerator System Advisory Committee) Review of the SNS Project, 2004.

[8] M. A. Plum, in SNS Injection Dump Beam Line Review, 2006.

[9] J. Error (private communication).

[10] OPERA-3D (an OPerating environment for Electromagnetic Research and Analysis) is the preprocessing and postprocessing system for electromagnetic analysis programs such as TOSCA (for nonlinear magnetostatic or electrostatic field and current flow problems) developed by Vector Fields Limited, England.

[11] J.G. Wang, in Proceedings of 18th Meeting of the International Collaboration on Advanced Neutron Sources, Dongguan, China, 2007; also see SNS-NOTEMAG-173, 2007.

[12] J.G. Wang, in Proceedings of the 2007 Particle Accelerator Conference, Albuquerque, NM, 2007, p. 3660; also see SNS-NOTE-MAG-178, 2007.

[13] J. Simkin, VF Inc. (private communication).

[14] J. G. Wang, Phys. Rev. ST Accel. Beams 9, 012401 (2006), http://prst-ab.aps.org/abstract/PRSTAB/v9/i1/e012401; also see SNS-NOTE-MAG-130, 2004.

[15] D. Raparia (SNS Collaboration), in Proceedings of the 2005 Particle Accelerator Conference, Knoxville, TN, p. 553.

[16] N. Tsoupas, in ASAC (Accelerator System Advisory Committee) Review of the SNS Project, 2002.

[17] J. G. Wang, SNS-NOTE-MAG-170, 2006.

[18] M. Reiser, Theory and Design of Charged Particle Beams (John Wiley \& Sons, Inc., New York, 1994), Chap. 5.

[19] N. Tsoupas, J. Brodowski, W. Meng, J. Wei, Y. Y. Lee, and J. Tuozzolo, in Proceedings of the 2002 European Particle Accelerator Conference, Paris (EPS, Paris, 2002), p. 1106. 
[20] N. Tsoupas, J. Jackson, Y. Y. Lee, D. Raparia, and J. Wei, in Proceedings of the 2003 Particle Accelerator Conference, Portland, Oregon, p. 2153.

[21] J. G. Wang, Phys. Rev. ST Accel. Beams 9, 122401 (2006), http://prst-ab.aps.org/abstract/PRSTAB/v9/i12/e0122401; also see SNS-NOTE-MAG-168, 2006.
[22] D. Raparia, Y. Y. Lee, J. Wei, and S. Henderson, in Proceedings of the 2003 Particle Accelerator Conference, Portland, Oregon, p. 3416.

[23] J. Holmes (private communication). 